\title{
PANDORA
}

\section{Plataforma de Administración de Objetos Reutilizables de Aprendizaje}

\section{Andrés Sucerquia Osorio}

Coordinador de diseno y desarrollo, univirtual,

Universidad Tecnologica de Pereira,

Carrera 27 \#10-02 Barrio Alamos - Pereira-Colombia sucerquia@utp.edu.co

Recibido: 1 Agosto 2013 - Revisado: 30 Septiembre 2013

Aceptado: 30 Octubre 2013 - Publicado: 30 Diciembre 2013

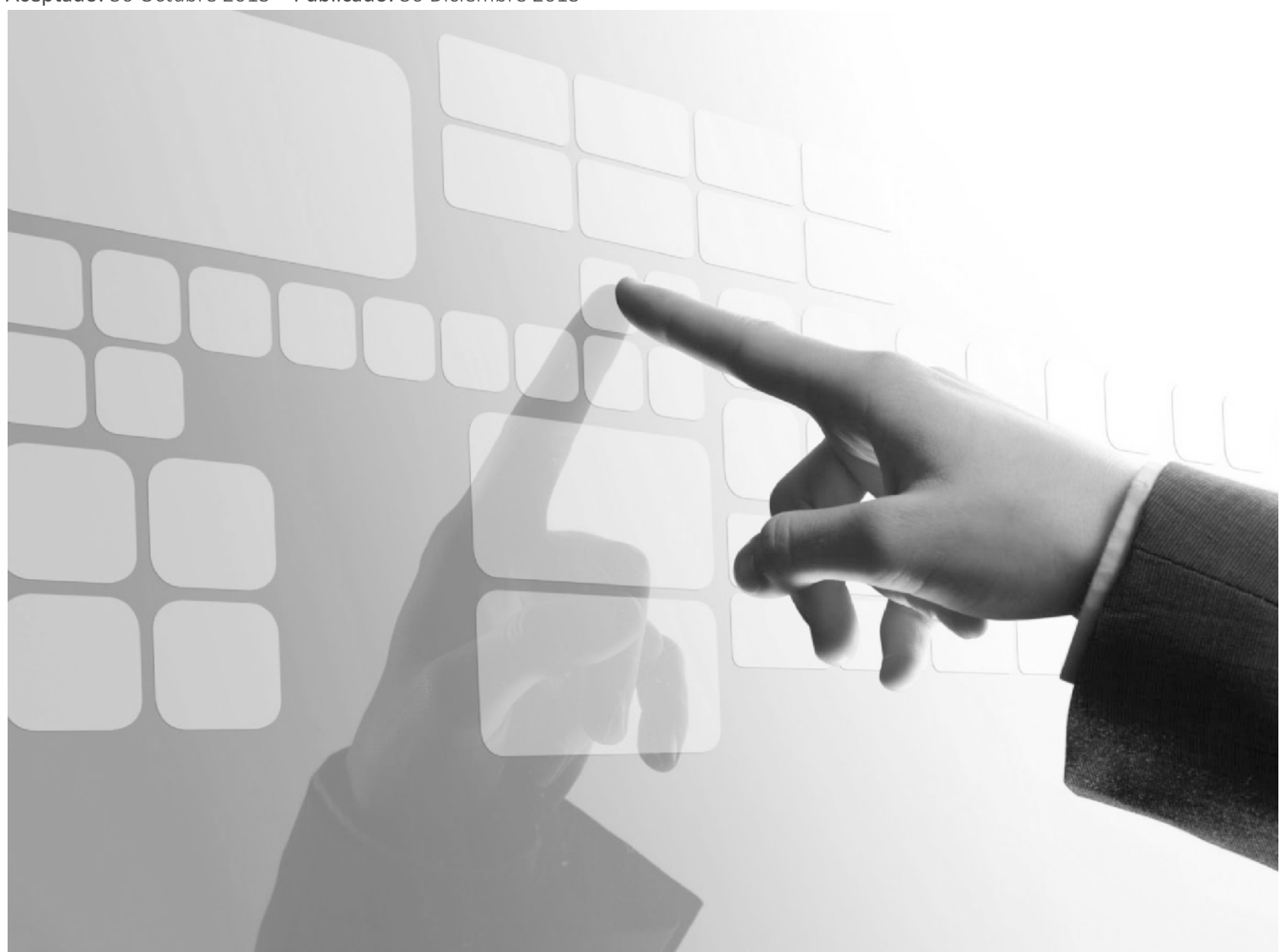

Resumen: Este documento presenta la experiencia de desarrollo e implementación de la plataforma de administración de objetos reutilizables de aprendizaje PANDORA, creada por la Universidad Tecnológica de Pereira en su dependencia de educación virtual Univirtual.

Palabras clave: Objetos de aprendizaje; sistemas distribuidos; sistemas de autor; SCORM; estándares e-learning.

Abstract: This paper presents the experience of developing and implementing of management platform reusable learning objects PANDORA, created by The Technological University of Pereira in their dependence on virtual education Univirtual.

Key words: Learning objects; distributed systems; authoring systems; SCORM; e-learning standards. 


\section{INTRODUCCIÓN}

Hoy en día, las empresas están en la obligación de tener sus procesos productivos de manera eficiente $y$ eficaz si quieren mantenerse vigentes en un mercado cada vez más competitivo y globalizado, por ende, la aplicación de herramientas que permitan obtener mejoras en sus procesos, solución a los problemas y satisfacción a los clientes, no se pueden obviar. La simulación precisamente, se utiliza para realizar representaciones de sistemas productivos y a la vez, poder estudiar cómo es el flujo de proceso; de esta manera,se permite realizar permutaciones con el objetivo de analizar el comportamiento y obtener una visión global de los cambios locales realizados en el sistema.

La simulación surge a partir de la evolución del Método de Montecarlo, gracias a la construcción de los primeros computadores de propósito general como el ENIAC; de igual forma, debido a la aplicación de modelos estadísticos y matemáticos por medio de herramientas informáticas. El desarrollo de este trabajo propone el uso del paquete de simulación ProModel, que posibilita construir y analizar el comportamiento del proceso y una de sus herramientas denominada Stat: Fit, con el fin de poder analizar los datos de entradas.

Ante el auge alcanzado por las herramientas, modelos y casos de aplicación en diversas áreas, surge la iniciativa de diseñar unas propuestas de mejora basados en el uso de la simulación.Para esto, en una segunda parte, se especifican los fundamentos teóricos e investigativos del proyecto; en una tercera instancia, los elementos metodológicos desarrollados; en una cuarta, el caso práctico de aplicación y, por último, las conclusiones.Andrés Sucerquia. PANDORA Plataforma de Administración de Objetos Reutilizables de Aprendizaje.

\section{HISTORIA}

En abril de 2008 se inicia el diseño del sistema, con el sustento teórico de la Mg. Martha Isabel Tobón Lindo y el Mg. Jorge Alberto Lozano Valencia, quienes conocían el trabajo hecho por el grupo de estudio de objetos de aprendizaje del MEN, y con el trabajo de los ingenieros de sistemas: Carlos Alberto López Giraldo, Juan Pablo Valencia Isaza, Andrés Sucerquia Osorio, Cesar Augusto Castaño Cuartas y el diseñador visual Sebastián Rozo Cadavid, se desarrolló la aplicación en dos fases, la primera fase se entregó en julio de 2009.

Para esta fase del desarrollo, se tomaron como referentes el portal educativo de Discovery http://www.discoveryeducation.com, el banco de objetos de aprendizaje del MEN http://64.76.190.172/drupalM/ y el repositorio de recursos ed ucativos Merlot http://www.merlot.org/merlot/index.htm. Asímismo se definió el uso del estándar SCORM (Sharable Content Object Reference Model) para empaquetar los objetos de aprendizaje producidos en la plataforma.

Para la segunda fase del desarrollo, se escaló la aplicación hacia una arquitectura distribuida, que a pesar de las complicaciones tecnológicas de comunicación y seguridad que surgieron para conectar los nodos de la red, potenciaba el alcance del proyecto haciendo que otras instituciones se vincularan a la red, diversificando y poblando la plataforma con recursos digitales y objetos de aprendizaje de forma colaborativa.

Para ello, se asumieron grandes desafíos tecnológicos, ya que ninguno de los integrantes del equipo tenía experiencia en el desarrollo de aplicaciones distribuidas, por lo tanto, se requirió de una etapa prolongada de estudio y pruebas de interoperabilidad del software. 
Como consecuencia de la interoperabilidad, fue necesario diseñar e implementar mecanismos de seguridad para proteger la comunicación entre los diferentes nodos, a través de mecanismos especializados de encriptación, autenticación y autorización. Se diseño un algoritmo de cifrado, para transmitir los mensajes entre los nodos.

La segunda fase del desarrollo de PANDORA se entregó en noviembre de 2010. Esta fase ya contaba con un instalador para los nodos y los mecanismos de comunicación implementados mediante Web Services tipo SOAP (Simple Object Access Protocol) usando XML (Extensible MarkupLanguage).

Posteriormente, se utilizó la plataforma de manera interna, realizando pruebas y ajustando los errores encontrados.

Dentro del marco del tercer encuentro presencial de la educación virtual Desconectado III realizado el 10 de noviembre de 2011, se presentó la ponencia llamada: "Llenando la Caja de PANDORA: Una propuesta para la construcción de objetos de aprendizaje reutilizables" (sucerquia\& palechor,2011) en la que se invitó a la comunidad educativa a utilizar la plataforma.

De igual forma, el 2 de diciembre de 2012 en el tercer ciclo de conferencias UNIVIRTUAL UNIVERSIDAD TECNOLÓGICA DE PEREIRA, se presentó la ponencia Ilamada "PANDORA: Una propuesta para complementar las prácticas educativas con el uso de TIC" (sucerquia\& cuartas, 2012) que trató sobre los usos pedagógicos de los objetos de aprendizaje.

\section{DESCRIPCIÓN DE PANDORA}

PANDORA es un sistema de gestión de contenidos, Learning Content Management Systems (LCMS), concebido, además, como un repositorio de recursos digitales y de objetos de aprendizaje reutilizables.
En PANDORA un objeto de aprendizaje reutilizable (en adelante OAR) es una entidad digital autocontenible, autónoma, reutilizable y evaluable, formada por los siguientes elementos:

- Objetivo de aprendizaje: Propósito educativo.

- Recursos multimedia: Contenidos educativos.

- Actividades de Aprendizaje: Pruebas Calificables.

- Instrucciones: Elementos de contextualización.

- Metadatos: Permiten su catalogación.

Ampliando la descripción de los elementos del OAR en PANDORA, se tiene:

(a) Objetivo de aprendizaje: Presenta de manera explícita lo que el estudiante aprenderá al utilizar el OAR.

(b) Recursos multimedia: Se refiere a los contenidos educativos digitales presentados por el OAR en cualquiera de los siguientes formatos:

- Documentos (htm, html, txt, rtf, pdf, doc, docx, pps, ppsx, ppt, pptx, $x|s, x| s x)$.

- Imágenes (gif,jpg, jpe, jpeg, bmp, png)

- Animaciones (swf).

- Audio (ra, ram, wav, mp3, wma, mid, midi).

- Video (flv, mpeg, mpg, mpe, avi, wmv, mov, mp4, ogg).

(c) Actividades de aprendizaje: Son dinámicas diseñadas por el autor del OAR para valorar la adquisición del objetivo de aprendizaje mediante una prueba. En PANDORA las actividades son automáticamente calificables empleando una escala de 0 a $100 \%$ en uno de los diferentes tipos de 


\section{Ingenierías: י}

actividades ofrecidas por la plataforma:

- Ordenamiento: Se plantea un interrogante que debe ser resuelto, ordenando los fragmentos del concepto que se está valorando.

- Emparejamiento: Se ofrece una columna de conceptos enfrentada a otra columna con las ampliaciones de los mismos en orden aleatorio, el propósito es relacionarlas aplicando la teoría presentada en los contenidos del OAR.

- Selección Múltiple: Se pueden construir cuestionarios de única o múltiple respuesta para poner en práctica los elementos asimilados del OAR.

- Falso o Verdadero: Se presenta un cuestionario con sentencias falsas o verdaderas acerca de los conceptos expuestos en el OAR.

(d) Instrucciones: Indicaciones claras y concisas que el autor incluye al construir los OAR para que estos sean consultados y utilizados correctamente en los diferentes contextos educativos donde pueden ser empleados.

(e) Metadatos: Una de las propiedades más importantes de los objetos de aprendizaje es la reutilización.

¿Cómo se puede lograr que los objetos de aprendizaje sean reutilizables? La respuesta a esta pregunta está en los metadatos (Wiley, 2006), que permiten que los Objetos de Aprendizaje se puedan compartir y reutilizar.

Un metadato es toda aquella información que permite identificar un Objeto de Aprendizaje.

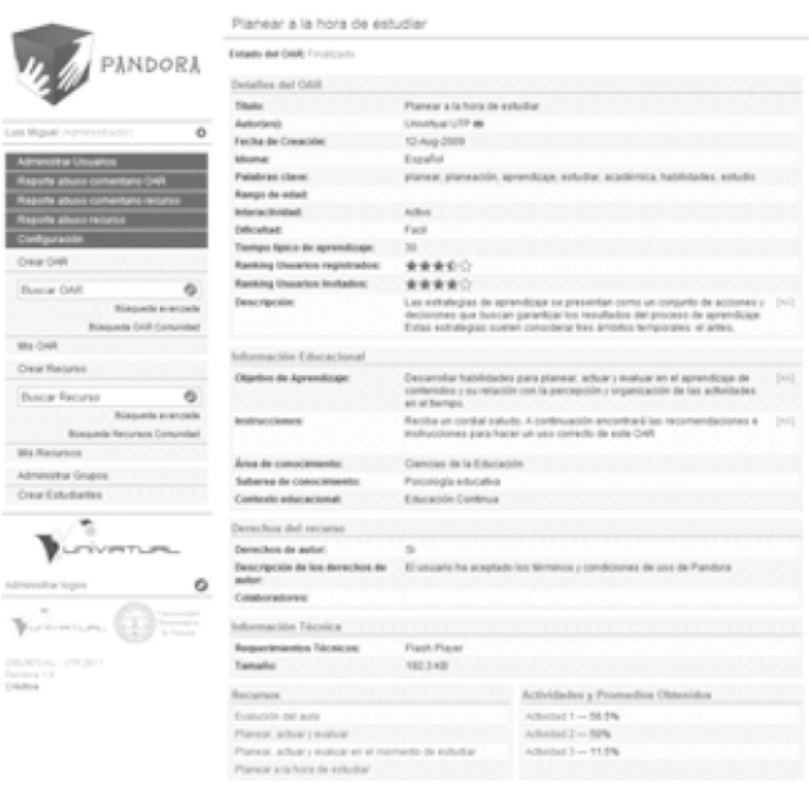

Figura 1. Metadatos de un OAR en PANDORA.

En la Fig. 1, se muestran algunos metadatos que posee un OAR en PANDORA.

En PANDORA se producen OAR que cumplen el estándar SCORM con los siguientes metadatos (ADL, 2006):

(a) General:

- Identifier: Identificador del OAR.

- Title:Título.

- Catalog (catálogo) = Para cumplir el estándar.

- Entry (entrada catálogo) = Para cumplir el estándar.

- Language: Idioma.

- Description: Descripción.

- Keyword: Palabras clave.

- Coverage (cobertura) = Para cumplir el estándar.

- Structure: (estructura) = Para cumplir el estándar.

- Aggregation Level: (agregación) = Para cumplir el estándar. 
(b) Life Cycle:

- Versión: Versión.

- Status: Estados (En construcción, Terminado, No disponible).

- Role: Rol de los colaboradores.

- Vcard: Datos de contacto tipo agenda electrónica.

- Date: Fecha de creación.

(c.) Technical:

- Format: Formato del OAR, en el caso de PANDORA son archivos descritos en el estándar como text/html.

- Size: Tamaño en kbytes.

- Location: URL del OAR.

- Requierements: Requerimientos técnicos para su correcto funcionamiento.

(d) Educational:

- Interactivitytype: Forma de interacción que ofrece el OAR, clasificada por el estándar como Activa, Expositiva, Mixta (una mezcla de las anteriores) o Indefinida.

- Learning Resource Type: Tipo de recurso.

- Interactivity level: Nivel interactividad.

- Semantic Density: Densidad semántica.

- Intendedenduser role: Por defecto en PANDORA es el estudiante.

- Context: Contexto educativo para el cual fue pensado el OAR, es decir, educación continua, pregrado, especialización, maestría, doctorado o posdoctorado.

- Typicalagerange: Rango de edad que recomienda el autor del OAR para los estudiantes que utilizarán el OAR.

- Difficulty: Grado de dificultad del OAR según el criterio del autor. Los grados pueden ser: muy fácil, fácil, medio, difícil o muy difícil.

- Typicallearning time: Cantidad de tiempo de aprendizaje estimado por el autor del OAR.

- Description: Descripción.

- Language: Idioma.

(e) Rights:

Cost: Se especifica si el uso del OAR requiere algún tipo de pago. En el caso de PANDORA es no por defecto, porque el uso de los OAR es gratuito.

\subsection{Características DE PANDORA}

Las características funcionales de PANDORA se listan a continuación:

(a) Gestión de recursos educativos digitales: Ofrece una interfaz que permite la creación, modificación, eliminación y búsqueda de contenidos digitales, como también la selección de recursos favoritos y propios.

(b) Gestión de OAR: Ofrece una interfaz que permite la creación, modificación, eliminación y búsqueda de objetos de aprendizaje, como también la selección de OAR favoritos y propios.

(c.) La creación de OAR hace uso de los recursos educativos digitales existentes en PANDORA y permite la construcción de actividades de aprendizaje.

(d) Se permite crear obras derivadas de un OAR mediante la opción de copia y queda referenciado el OAR original.

(e) Se permite la generación del paquete SCORM de un OAR para ser usado en LMS (Learning Management System) que soporten el estándar.

(f) Gestión de grupos: Permite gestionar listas de estudiantes con correos electrónicos, para envío de OAR.

\section{(g) Distribución:}

Permite compartir los OAR enviando un enlace vía correo electrónico a una o muchas listas de distribución.

- Permite compartir el OAR a través de una URL simple, que puede ser enlazada desde una página web.

- Presenta el resultado de las calificaciones del OAR cuando este fue configurado para almacenarlas. Los resultados podrán ser obtenidos en una interfaz o en un archivo en formato csv para su uso personal. 
(h) Elementos de inteligencia colectiva:

- Comentarios: pueden ser hechos anónimamente o con el usuario registrado en la plataforma.

- Contador de uso: lleva el registro de cuantas veces es usado el OAR (se entiende por usar el OAR, a la acción de completar las actividades de aprendizaje del mismo).

- Ranking: permite a usuarios registrados y anónimos, calificar los recursos y OAR usando la escala de 5 estrellas.

- Reporte de abuso: permite reportar cualquier abuso en los OAR, recursos y comentarios.

- OAR relacionados: recomienda otros OAR que tengan relación con el OAR actual.

-

\section{RESULTADOS OBTENIDOS}

Se han usado objetos de aprendizaje construidos en PANDORA en el proceso de formación virtual Ilamado "Ruta de Formación Docente para el uso y apropiación de las Tecnologías de la Información y la Comunicación (TIC) en los procesos de enseñanza y aprendizaje" (Univirtual, 2011), que está basado en el libro Formación Continua para Docentes UTP (Tobón, 2010). La ruta de formación docente contó con la participación de 200 docentes de la Universidad Tecnológica de Pereira y se certificaron dos niveles, el primero de 100 horas y el segundo, de 80 horas.

Los OAR utilizados en la Ruta de Formación Docente fueron empaquetados en formato SCORM y luego publicados en el LMS moodle.

Gracias a que los OAR de PANDORA envían la calificación de sus actividades al libro de calificaciones del LMS de forma automática, brindan un criterio de valoración útil sobre el logro del objetivo de aprendizaje de cada estudiante.

\section{CONCLUSIONES}

El potencial de PANDORA se encuentra en el uso que se haga de ella. Por ejemplo, si se tiene un curso de
Introducción a la Informática con 40 estudiantes, se puede crear un objeto de aprendizaje compuesto por recursos educativos que presenten la historia y expliquen el funcionamiento de los computadores, estos recursos pueden ser animaciones html5, videos, imágenes, PDF; así, se elaboran actividades de retroalimentación que indaguen sobre los contenidos desarrollados en el objeto de aprendizaje. Además, se puede crear una lista de distribución con los 40 correos de los estudiantes y compartirles el objeto de aprendizaje indicando que se quiere recibir la calificación de todos los intentos de cada alumno. De esta forma, el docente puede saber quiénes usaron el objeto de aprendizaje, cuántos intentos y qué calificación obtuvieron en cada intento.

También, puede compartir el enlace del objeto de aprendizaje para que cualquier persona lo use sin que las calificaciones se guarden. Y la última posibilidad es usar el objeto de aprendizaje integrado en un LMS gracias al paquete SCORM.

Los objetos de aprendizaje en PANDORA respetan el principio de autocontenibilidad, es decir, todos sus recursos educativos y actividades de aprendizaje están contenidos en el objeto mismo, sin depender de recursos externos. Así, permite su portabilidad mediante paquete SCORM y reutilización, mediante copia para obra derivada.

Se puede explorar la herramienta con los siguientes datos de acceso:

\section{URL:http://univirtual.utp.edu.co/pandora \\ Usuario: teknos \\ Contraseña: teknos}

Trabajo Futuro Experimentar con las capacidades que ofrece HTML5 para la reproducción multimedia sin la necesidad de instalar software adicional (codecs) a un navegador web moderno, las animaciones y transiciones CSS3, el 3D con WebGL, y la interactividad que se puede lograr con el buen uso de lenguaje Javascript. 
Se hace necesario implementar HTML5 en PANDORA, conjuntamente con diseño adaptable (ResponsiveDesing), para que la plataforma se visualice adecuadamente en los diferentes dispositivos y a diferentes resoluciones de pantalla.

Igualmente, se espera que PANDORA sea utilizado masivamente por docentes y estudiantes en cualquier contexto educativo en Colombia y en otros países que estén interesados en hacer parte de la comunidad PANDORA.

Agradecimientos A todo el equipo de Univirtual: Director, Jorge Alberto Lozano Valencia; coordinador general, Andrés Felipe Muñoz Ramírez; Andrés Oswaldo Palechor Ocampo; Vanessa Cataño Gamboa; Luis Miguel Escobar; Sebastián Rozo Cadavid; Juan Pablo Valencia Isaza; César Augusto Castaño Cuartas; Carlos Alberto López; María del Carmen Falcón y muy especialmente, a la ingeniera Martha Isabel Tobón Lindo.

\section{REFERENCIAS}

ADL. (2006). SCORM-2004 Sharable Content Object Reference Model Run-Time Environment (3a Edition). Version 1.0. Advanced Distributed Learning. Recuperado de http://www.adlnet.gov/capabilities/scorm/scorm-20043rd

ADL. (2006). SCORM-2004 Sharable Content Object Reference Model Content Aggregation Model (3a Edition). Version 1.0. Advanced Distributed Learning. Recuperado de http://www.adlnet.gov/capabilities/scorm/scorm-20043rd

ADL. (2006). SCORM-2004 Sharable Content Object Reference Model Impact Summary (3a Edition) Version 1.0. Advanced Distributed Learning. Recuperado de h t t p : / / w w w a d I n e t. gov/ w p content/uploads/2011/07/scorm2004-3rd ImpactSummary.pdf

ADL. (2006). SCORM-2004 Sharable Content Object Reference Model Overview (3a Edition). Version 1.0. Advanced Distributed Learning . Recuperado de http://www.adlnet.gov/capabilities/scorm/scorm-20143rd

ADL. (2006). SCORM-2004 Sharable Content Object Reference Model Sequencing and Navigation (3a Edition) Version 1.0. Advanced Distributed Learning. Recuperado de http://www.adlnet.gov/capabilities/scorm/scorm-20143rd

Alva, G. (2009). Sistemas Distribuidos: Seguridad y Protección. Recuperado de http://es.scribd.com/doc/14960477/Seguridad-yproteccion-en-Sistemas-Distribuidos

APROA. (2005). Manual de buenas prácticas para el desarrollo de objetos de aprendizaje. Recuperado de http://formacionprofesional.homestead.com/Objetos deaprendizaje.pdf

Apshankar, K. (2002). Professional open source web services. WroxPress.

Chiape, A., Cifuentes, Y. S., y Rodríguez, H. Y. R. (2007). Toward an instructional design model based on learning 
objects. Educational Technology Research and Development. Recuperado de http://www.springerlink.com/content/u84w63873vq77h2 h

Hanley, G., Reisman, S., Swift, C., y Moss, P. (s.f.). MERLOT. Recuperado de http:/www.merlot.org/merlot/index.htm

Lacasa, P., Vélez, R., y Sánchez, S. (2004). Objetos de aprendizaje y significado. Recuperado de

http//www.spdece.uah.es/papers/Lacasa Final.pdf

Palechor A. (2012). Tercer cliclo de conferencias Univirtual Universidad Tecnológica de Pereira. Recuperado de http//www.youtube.com/watch?v=hOnDSzerkRAO

Sucerquia, A. Palechor A. (2011). Desconectado III Encuentro Presencial de la Educación Virtual. Pereira. Recuperado de

http//www.youtube.com/watch?v=EwQ_8SabCTM

Sucerquia, A., Castaño C. (2012). Una mirada desde lo tecnológico a la Construcción de Objetos de Aprendizaje con PANDORA. Recuperado de:

https://www.youtube.com/watch?v=2tzmxzy55Ss

Tobón, M. (2007). Diseño Instruccional en un entorno de Aprendizaje Abierto. Pereira: Universidad Tecnológica de Pereira Univirtual. Recuperado de

http://opendata.socrata.com/views/g954-2ypq/obsolet files/6064d716-c4f0-4c57-ad7b-2ccd091bebbb

Tobón, M., Arbelaez, M., Falcón, M. y Bedoya, R. (2010). La Formación Docente al Incorporar las TIC En Los Procesos de Enseñanza y Aprendizaje, Una propuesta para la Universidad. Recuperado de

https://univirtual.utp.edu.co/portal/archivos_nota/16531 6_laformaciondocente.pdf

Univirtual, UTP. (2011). Ruta de formación Docente en TIC. Recuperado de

https://univirtual.utp.edu.co/portal/archivos_nota/23142 induccion_ruta_de_formacion_docente_utp.pdf

Wiley, D. (2001). Connecting learning objects to instructional design theory: A definition, a metaphor, and a taxonomy. Recuperado de

http://www.reusability.org/read/chapters/wiley.doc
Wiley, D. (2006). RIP-ping on Learning Objects. Recuperado de http://opencontent.org/blog/archives/230

Wiley, D. (2001). The Instructional Use of Learning Objects. Recuperado de http://reusability.org/read/

W3C, \&Swick, R. (2002). Metadata Activity Statement. Recuperado de

http://www.w3.org/Metadata/Activity.html

Zapata, M. (2005). Secuenciación de contenidos y objetos de aprendizaje. Recuperado de http://www.um.es/ead/red/M2/zapata47.pdf 